International Journal of Modern Physics: Conference Series

(C) World Scientific Publishing Company

\title{
MULTIWAVELENGTH PROBES OF THE ENVIRONS OF RELATIVISTIC SHOCKS IN BLAZAR JETS
}

\author{
MATTHEW G. BARING \\ Department of Physics and Astronomy - MS 108, Rice University, \\ 6100 Main Street, Houston, Texas 77251-1892, USA \\ baring@rice.edu \\ MARKUS BÖTTCHER \\ Centre for Space Research, North-West University, \\ Potchefstroom Campus, Potchefstroom, 2520, South Africa \\ Markus.Bottcher@nwu.ac.za \\ ERROL J. SUMMERLIN \\ Heliospheric Physics Laboratory, Code 672, \\ NASA's Goddard Space Flight Center, Greenbelt, MD 20770, USA \\ errol.summerlin@nasa.gov
}

Received October 29, 2021

Revised October 29, 2021

\begin{abstract}
Diffusive shock acceleration (DSA) at relativistic shocks is likely to be an important acceleration mechanism in various astrophysical jet sources, including radio-loud AGN. An important recent development for blazar science is the ability of Fermi-LAT data to pin down the power-law index of the high energy portion of emission in these sources, and therefore also the index of the underlying non-thermal particle population. This diagnostic potential was not possible prior to Fermi launch, when gamma-ray information was dominated by the highly-absorbed $\mathrm{TeV}$ band. This paper highlights how multiwavelength spectra including X-ray band and Fermi data can be used to probe diffusive acceleration in relativistic, oblique, MHD shocks in blazar jets. The spectral index of the non-thermal particle distributions resulting from Monte Carlo simulations of DSA, and the fraction of thermal particles accelerated to non-thermal energies, depend sensitively on the particles' mean free path scale, and also on the magnetic field obliquity to the shock normal. We investigate self-consistently the radiative synchrotron/Compton signatures of the resulting thermal and non-thermal particle distributions. Important constraints on the frequency of particle scattering and the level of field turbulence are identified for the blazar AO $0235+164$. The possible interpretation that turbulence levels decline with remoteness from jet shocks, and a significant role for non-gyroresonant diffusion, are discussed.
\end{abstract}

Keywords: Blazars, relativistic jets, shock acceleration, MHD turbulence, diffusion, Xrays, gamma-rays.

PACS numbers: 52.27.Ny, 52.35.Tc, 52.35.Ra, 97.80.Jp, 95.85.Pw, 98.54.Cm, 98.62.Nx 


\section{Introduction}

Blazars are a class of active galaxies that are among the most powerful and dynamic extragalactic objects. They exhibit rapid flares in radio, optical, X-ray and $\gamma$-ray wavebands. The general absence of emission lines in their optical spectra, originally the identifying feature of $\mathrm{Bl}$ Lac objects like BL Lacertae, together with detections of polarization in both radio and optical bands, suggests that the radio-to-optical continuum is non-thermal synchrotron radiation. In many blazars, this component extends to X-rays. The watershed development that isolated the class of blazars was the discovery ${ }^{112}$ of transient gamma-ray emission in 3C 279 and Mrk 421 by the EGRET instrument on the Compton Gamma-Ray Observatory at around 100 $\mathrm{MeV}-1 \mathrm{GeV}$. This was almost contemporaneous with the determination that Mrk 421 also emitted at $\mathrm{TeV}$ energies ${ }^{3}$. The prevailing thought is that this gammaray signal is generated by inverse Compton scattering of either an IR/optical/UV photon source of possibly disk or ambient origin ${ }^{45}$, or of synchrotron photons by the same electrons that emit this radio-to-X-ray signal, so-called synchrotron-selfCompton (SSC) model 66 6/78. An important recent development for blazar science has been the improvement of sensivity in the $100 \mathrm{MeV}-100 \mathrm{GeV}$ window, afforded by the Fermi-Large Area Telescope (LAT). Over the last five years, LAT data has enabled measurements of the power-law index of blazar spectra ${ }^{910}$, and therefore also the index of the underlying non-thermal particle population.

The flux variability and enormous power of blazars indicate that they possess central supermassive black holes with masses typically above $10^{8} M_{\odot}$, and are characterized by powerful jets that emanate from near the event horizon and point almost directly towards Earth. The rapid variability seen in $\mathrm{GeV}-\mathrm{TeV}$ flares drives the prevailing picture for the blazar environment: their jets are relativistic, and compactly structured on small spatial scales that are unresolvable by present gamma-ray telescopes. Velocity structure in the supersonic outflow in these jets naturally generates relativistic shocks, and these can form the principal sites for acceleration of electrons and perhaps ions to the ultrarelativistic energies demanded by the X-ray and $\gamma$-ray data ${ }^{11}$. Diffusive acceleration at such shocks is believed to be the main mechanism for energizing such charges $11|12| 13$. This is because it is both extremely efficient and very fast, precipitating acceleration rates $\dot{\gamma}$ of the order of the gyrofrequency $\omega_{g}=e B / m c$, since the diffusive collisions of electrons and ions with MHD turbulence in jets is usually dominated by gyroresonant interactions. The diffusive mean free path $\lambda$ of a charge is then at least comparable to its Larmor radius $r_{g}=p c / e B=\gamma \beta m c^{2} / e B$ if it has a momentum $p=\gamma \beta m c$ and Lorentz factor $\gamma=1 / \sqrt{1-\beta^{2}}$; the $\lambda \sim r_{g}$ regime is known as the Bohm limit. This scenario invokes a conversion of the ballistic kinetic energies of colliding, large-scale MHD structures in jets into the energization of $\gamma \gg 1$ particles at relativistic shocks. Other paradigms such as acceleration by reconnection of magnetic fields embedded in Poynting-flux dominated outflows can be envisaged. Yet, there is presently no compelling reason to disavow the shock acceleration picture for blazar jets. 
To explore the shock paradigm, the standard practice has been to develop multiwavelength (MW) spectral models, spanning radio to $\mathrm{TeV}$ gamma-ray wavelengths $4|5| 6|7 / 8| 14$. This generally gives a broad-brush assessment of a range of jet environment parameters, but the spectral fits in any one band are of limited quality. The high statistics spectroscopy offered by the Fermi-LAT data demands a closer look at the constraints observations can make on the shock acceleration process and the blazar jet environment. For example, it has long been known that non-relativistic shocks generate a power-law index that is dependent only on the hydrodynamic velocity compression ratio $r$ across the shock ${ }^{12 \mid 13}$. For strong shocks with $r=4$, this yields a $p^{-2}$ distribution. This robustness has helped underpin a consensus that Galactic cosmic rays originate in supernova remnants. In contrast, the convective loss rates downstream of relativistic shocks are sensitive to the orientation of the mean magnetic field, and the character of the in situ MHD turbulence, yielding a wide variety of power-law indices for accelerated charges for a given velocity compression ratis $15|16| 17 \mid 18$. Accordingly, gamma-ray spectroscopy of blazars can afford insights into details of the structure of shocks in jets. In addition, it has been understood for nearly two decades ${ }^{19}$ that diffusive shock acceleration is so efficient that low levels of field turbulence (spawning $\lambda \gg r_{g}$ ) are required to accommodate synchrotron spectral peaks appearing in the X-ray band.

In gleaning such insights, multi-wavelength blazar models generally invoke an elemental description of the distribution of accelerated charges, usually a powerlaw truncated at some minimum particle Lorentz factor. Such forms omit the hot Maxwellian-like components that naturally arise due to shock layer turbulent dissipation of the bulk kinetic energy of the upstream flow. This paper goes beyond such simplified approaches by employing detailed results from comprehensive Monte Carlo simulations of diffusive acceleration at relativistic shocks, building upon the

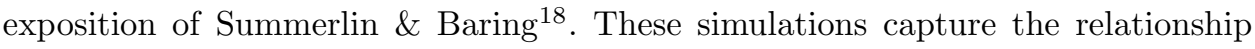
between turbulence parameters and the power-law index, and also the connection between the thermal bulk of the population and the power-law tail of the accelerated species. We fold these results through the one-zone SSC/external Compton models of Böttcher et al ${ }^{20}$ for radiation emission and transport in blazars. In particular, diagnostics are obtained for the frequency of particle scattering and the level of field turbulence for the $\mathrm{Bl}$ Lac object $\mathrm{AO} 0235+164$. By exploring a range of dependences of the diffusive mean free path $\lambda$ on the momentum $p$ of accelerated charges, the possible interpretation that turbulence levels decline with remoteness from a shock is identified, perhaps signalling a significant role for non-gyroresonant diffusion near blazar jet shocks. 


\section{Shock Acceleration Simulations of Particle Distributions}

In collisionless shocks, non-thermal, charged particles gain energy by scattering between MHD turbulence that is "anchored" in the converging upstream and downstream plasmas. In non-relativistic shocks, the energetic particles are nearly isotropic in these fluid frames, since their speeds $v \approx c$ far exceed $u_{1 x}\left(u_{2 x}\right)$, the upstream (downstream) flow speed component in the co-ordinate direction $x$ normal to the shock. The acceleration process possesses no momentum scale, and the resulting distribution takes the form $d n / d p \propto p^{-\sigma}$, where $\sigma=(r+2) /(r-1)$, for $r=u_{1 x} / u_{2 x}$ being the shock's velocity compression ratio. The index $\sigma$ in this limit is independent of the shock speed, $u_{1}$, the upstream field obliquity angle $\Theta_{\mathrm{Bf} 1}$ (to the shock normal), and any details of the scattering process. In this test-particle limit, high Mach number non-relativistic shocks have $r \simeq 4$ yielding $d n / d p \propto p^{-2}$.

In contrast, in relativistic shocks, plasma anisotropy is prevalent, and $\sigma$ becomes a function of the flow speed $u_{1}$, the field obliquity, and the nature of the scattering. Test-particle acceleration in parallel relativistic shocks evinces the key property that a so-called "universal" spectral index ${ }^{21|22| 23}, \sigma \sim 2.23$ exists in the two limits of $\Gamma_{1} \gg 1$ and small angle scattering, i.e., $\delta \theta \ll 1 / \Gamma_{1}$, for a shock compression ratio of $r=3$. Here $\delta \theta$ is the average angle a particle's momentum vector deviates in a scattering event, and $\Gamma_{1}=\left(1-\left[u_{1} / c\right]^{2}\right)^{-1 / 2}$ is the Lorentz factor of the upstream flow in the shock rest frame. For all other parameter regimes in relativistic shocks, large departures from this special index are observed. Note that relativistic shocks in blazar environs are typically highly oblique $\left(\Theta_{\mathrm{Bf} 1} \gtrsim 1 / \Gamma_{1}\right)$ due to the Lorentz transformation of ambient, upstream magnetic fields to the shock rest frame.

Various approaches have been adopted to model diffusive shock acceleration (DSA) at relativistic discontinuities. These include analytic methods $\$ 24|25| 23$, and Monte Carlo simulations of convection and diffusion 15|21|17|26|18. Particle-in-cell (PIC) plasma simulations have also been employed to study this problem via modeling the establishment of turbulence self-consistently $27|28| 29$, though they cannot yet explore acceleration beyond modest non-thermal energies. The Monte Carlo approach is ideal for describing the diffusive acceleration of particles from thermal (injection) scales out to the highest energies relevant to blazar jet models.

The Monte Carlo technique employed here solves the Boltzmann equation with a phenomenological scattering operator $\underline{13|15| 18}$. The simulation space is divided into grid sections with different flow speeds, magnetic fields, etc. For electron-ion shocks, the vastly different inertial scales can be easily accommodated. Particles are injected far upstream and diffuse and convect in the shock neighborhood. Their flux-weighted contributions to the momentum distribution function are logged at any position $x$. Each particle is followed until it leaves the system by either convecting far downstream, or exceeding some prescribed maximum momentum $p_{\max }$.

The details of particle transport are given in 15|17|18: the simulation can model both scatterings with small angular deflections $\delta \theta \ll 1 / \Gamma_{1}$ (seeding pitch angle diffusion) and large ones $\left(\delta \theta \gtrsim 1 / \Gamma_{1}\right)$ using several parameters. In this paper, 
the focus will be on $\delta \theta \ll 1 / \Gamma_{1}$ domains; results for large angle scattering are illustrated in $15 \mid 30$. The simulation assumes that the complicated plasma physics of wave-particle interactions can be described by a simple scattering relation for individual particles, viz.

$$
\lambda_{\|}=\lambda_{1} \frac{\rho_{1}}{\rho}\left(\frac{r_{g}}{r_{g 1}}\right)^{\alpha} \equiv \eta_{1}\left(\frac{p}{p_{1}}\right)^{\alpha} \quad \text { and } \quad \kappa_{\|}=\lambda_{\|} v / 3,
$$

where $v=p / m$ is the particle speed in the local upstream or downstream fluid frame, $r_{g}=p c /(Q e B)$ is the gyroradius of a particle of charge $Q e$, and $\rho$ is the plasma density, with a far upstream value of $\rho_{1}$. Here, $\lambda_{\|}\left(\kappa_{\|}\right)$is the mean free path (spatial diffusion coefficient) in the local fluid frame, parallel to the field $\mathbf{B}$, with $\lambda_{\|} \gtrsim r_{g}$ being a fundamental bound (Bohm limit) for physically meaningful diffusion. Scalings of the momentum $p_{1}=m u_{1 x}=m \beta_{1 x} c$ and the mean free path $\lambda_{1}=\eta_{1} r_{g 1}$ for $r_{g 1}=p_{1} c /\left(Q e B_{1}\right)$ are introduced to simplify the algebra.

Scattering according to Eq. [1] is equivalent to a kinetic theory description 31 where the diffusion coefficients perpendicular to $\left(\kappa_{\perp}\right)$ and parallel to $\left(\kappa_{\|}\right) \mathbf{B}$ are related via $\kappa_{\perp}=\kappa_{\|} /\left[1+\left(\lambda_{\|} / r_{g}\right)^{2}\right]$. The parameter $\eta \equiv \lambda_{\|} / r_{g}$ then characterizes the "strength" of the scattering and the importance of cross-field diffusion: when $\eta \sim 1$ at the Bohm Limit, $\kappa_{\perp} \sim \kappa_{\|}$and particles diffuse across magnetic field lines nearly as quickly as along them. Each scattering event is an elastic deflection of the fluid frame momentum vector $\mathbf{p}$ through angle $\sim \delta \theta$, so that the number of deflections constituting $\lambda_{\|}$, a large angle deflection scale, is proportional to $(\delta \theta)^{-2}$.

Observations at the Earth's bow shock ${ }^{32}$ indicate that $1 / 2<\alpha<3 / 2$, while $\alpha \sim 1 / 2$ is obtained from turbulence in the interplanetary magnetic field $\frac{33}{\text {. Hy- }}$ brid plasma simulations 34 suggest a mean free path obeying Eq. 1 with $\alpha \sim 2 / 3$. Within the confines of quasi-linear theory of MHD turbulence, diffusion characteristics embodied in Eq. (1) can be coupled ${ }^{31}$ to the inertial range and power spectrum $\langle\delta B / B\rangle^{2}$ of wave numbers $\mathbf{k}$ for the turbulence. These solar wind observations and plasma simulation results explore domains where the scales of turbulence $\mathbf{k}$ and diffusion $\lambda_{\|}$are very restricted. The story that will unfold in this paper is that blazar jets may present a very different picture with their associated large dynamic ranges of momenta and mean free paths in relativistic jets.

In relativistic shocks, the distribution functions of accelerated charges are sensitive to the choices of both the $\eta_{1}=\lambda_{1} / r_{g 1}$ and $\alpha$ diffusion parameters. The $\eta$ dependence of acceleration is illustrated at length in Summerlin \& Baring ${ }^{18}$, where $\alpha=1$ is a restriction imposed for simplicity, so that $\lambda_{\|} \propto r_{g}$. In subluminal shocks, those where a de Hoffman-Teller (HT) frame ${ }^{\sqrt{35}}$ can be found (i.e., $u_{1 x} / \cos \Theta_{\mathrm{Bf} 1}<c$, where again, $\Theta_{\mathrm{Bf} 1}$ is the upstream field obliquity angle to the shock normal), distributions $d n / d p \propto p^{-\sigma}$ generally possess indices in the range $1<\sigma<2.5$. The HT frame is where the shock is at rest and the fluid flows along B. An example of such a spectrum is the $\eta=10$ case in Fig. 11, where the power-law tail smoothly blends into the upper end of the thermal distribution. For much of the fairly restricted range of obliquities corresponding to $u_{1} / \cos \Theta_{\mathrm{Bf} 1}<c$, the larger the value of $\eta$, 


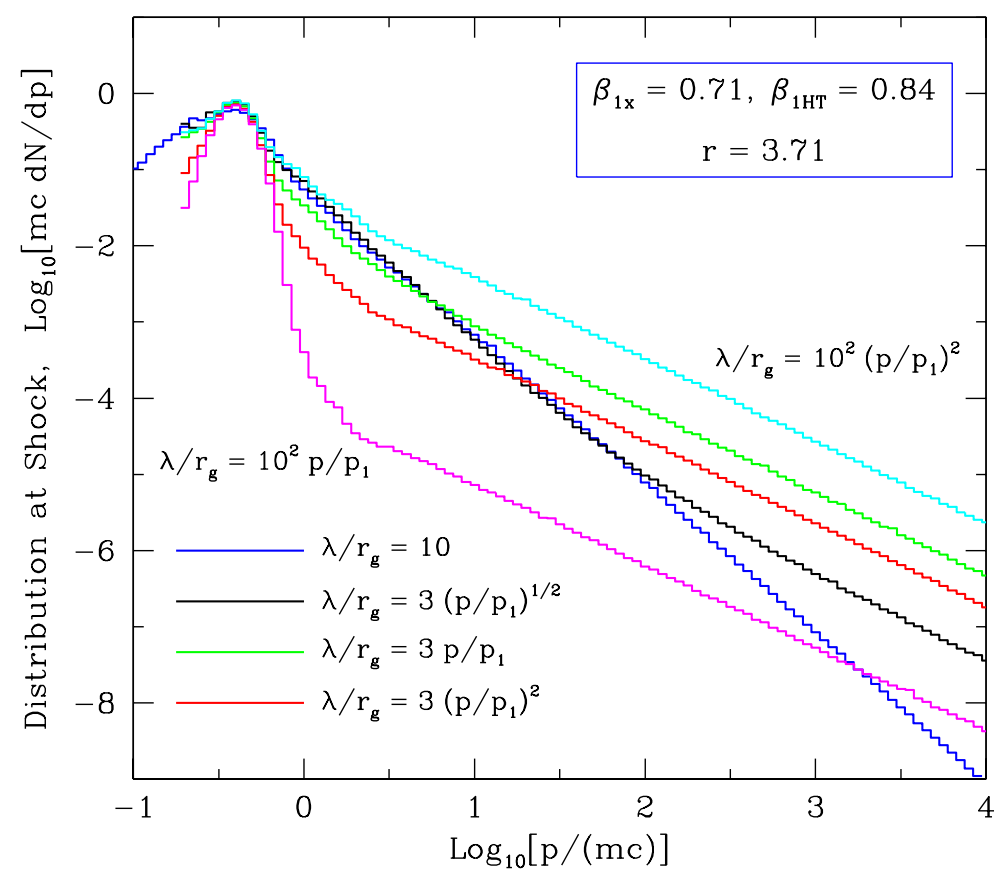

Fig. 1. Particle distributions for acceleration simulation runs in the small angle scattering limit, for strong mildly-relativistic shocks of upstream flow speed $\beta_{1 x} \equiv u_{1 x} / c=0.71$. Here the de Hoffmann-Teller frame upstream flow speed was set at $\beta_{1 \mathrm{HT}}=0.75=\beta_{1 x} / \cos \Theta_{\mathrm{Bf} 1}$, with $\Theta_{\mathrm{Bf} 1} \approx$ $48.2^{\circ}$ being the upstream field obliquity to the shock normal. Distributions are displayed for six different forms for the momentum dependence of the diffusive mean fee path $\lambda \equiv \lambda_{\|}$, namely $\lambda / r_{g} \propto p^{\alpha-1}$ with $\alpha-1=0,1 / 2,1$ and 2, as labelled - see Eq. 1 1 and associated discussion. The shock velocity compression ratio was fixed at $r=u_{1 x} / u_{2 x}=3.71$, and the upstream temperature corresponded to a sonic Mach number of $M_{\mathrm{S}} \sim 4$. See Fig. 10 of Ref. [18] for more $\alpha=1$ cases.

the lower $\sigma$ is. In fact, when $\eta \gg 1, \sigma \sim 1$ is generally observed $\frac{18}{18}$ and the acceleration distribution is extremely flat. The origin of this behavior was found to be shock drift acceleration, where charges with select gyrational phases incident upon the shock from upstream, are trapped and reflected by the shock. Shock drift acceleration is a phenomenon that has been well-studied in the context of heliospheric shocks $56 \mid 37$. Successive episodes of upstream excursions and reflection at the shock precipitate efficient acceleration and postpone convective loss downstream for many shock interaction cycles. Only when the field obliquity $\Theta_{\mathrm{Bf} 1}$ is high enough that the shock is superluminal and $u_{1} / \cos \Theta_{\mathrm{Bf} 1}>c$ does the powerful convective action of the flow overwhelm reflection, and the acceleration process effectively shuts of ${ }^{17}[18 \mid 38$, with the index $\sigma$ increasing rapidly above $3-4$.

Here, these results are extended to situations where $\eta(p)$ is an increasing function of momentum $p$. The simplest prescription that captures such character is Eq. (1), and representative $\alpha>1$ examples are displayed in Fig. 1. The subluminal 
shock parameters used therein are mostly those in Fig. 10, left panel, of Ref. [18]. The distributions all display the generic trait of a dominant thermal population with a power-law tail that extends as high as the geometric scale of the diffusive acceleration zone permits: this environmental parameter is addressed in Section 3 . Each distribution possesses an injection efficiency from thermal into the Fermi process that reflects the value of $\eta_{1}$ in Eq. (1). Large values of $\eta_{1}$ produce a low normalization to the power-law at supra-thermal momenta $p \sim 3 p_{1}-10 p_{1}$. The ultimate power-law index at high momenta $p \gg p_{1}$ is determined by large values for $\eta(p)$ in all these simulation runs, i.e., realizing $\sigma \sim 1$ regimes in weak turbulence in Fig. 1 due to efficient shock drift acceleration. The key new signature presented here is the appearance of "flattening" breaks in the high energy tail that are manifested when $\eta(p)$ begins to exceed values around 10-30; see the $\eta_{1}=3, \alpha=3 / 2$ and $\eta_{1}=3$, $\alpha=2$ cases in Fig. 1. Hence, $\alpha>1$ models can possess relatively inefficient injection at thermal energies, with very dominant thermal components, but exhibit efficient acceleration at momenta $p \gtrsim 100 p_{1}$. The properties of plasma turbulence that might generate $\alpha>1$ circumstances are discussed below in Section 4

\section{Radiation Emission Modeling}

The focus now turns to modeling the broadband continuum emission of the blazar AO 0235+164 using specific distributions generated by the Monte Carlo technique. The objective is to assess how the global character of the radio to X-ray to gammaray data can provide insights into the nature of the accelerator and its plasma environment. To facilitate this goal, we focus solely on leptonic models $6 / 7|8| 20$, those with synchrotron emission predominantly at frequencies below $10^{19} \mathrm{~Hz}$, and with inverse Compton emission dominating the gamma-ray signal. Hadronic models are also of great interest, but then introduce more parameters, and so don't tend to provide constraints that are as restrictive as those explored here.

To evaluate the emission from the Monte Carlo generated electron distributions described in the previous section, we adopt radiation modules from the one zone blazar radiation transfer code of Böttcher et al 20039 . This code treats synchrotron emission, and also inverse Compton scattering of both synchrotron and external seed photons. Bremsstrahlung is modeled in the code, but is usually insignificant in blazars: it is so for AO $0235+164$. For this purpose, we first note that without the introduction of information on the physical scale of the acceleration zone, the Monte Carlo simulations do not describe the high-energy cut-off of the electron distribution. We therefore extend the initial electron spectra like those in Fig. 1 out to a maximum energy $\gamma_{\max } m c^{2} \approx p_{\max } c$. Note that such an extrapolation is acceptable because the asymptotic power-law has been realized in the Monte Carlo simulations. This maximum energy (a model parameter) is constrained in two ways: (a) particles will not be accelerated beyond an energy for which the (synchrotron plus inverse Compton) radiative cooling time scale, $t_{\mathrm{rad}}=3 m_{e} c^{2} /\left(4 c \sigma_{\mathrm{T}} \mathcal{U} \gamma\right)$, where $\mathcal{U}=\mathcal{U}_{B}+\mathcal{U}_{\text {rad }}$ is the sum of the energy densities in the magnetic field and the 
photon field, is shorter than the acceleration time scale, $t_{\mathrm{acc}}=\gamma m_{e} c \eta(p) /(e B)$; and (b) particles will not be accelerated to energies at which the diffusive mean free path $\lambda_{\|}=\eta(p) r_{g}(\gamma) \equiv \eta_{1}\left(p / p_{1}\right)^{\alpha}$ in Eq. (1) exceeds the size $R_{\text {acc }}$ of the acceleration zone. Here $\gamma=\left\{1+(p / m c)^{2}\right\}^{1 / 2} \approx p /(m c)$ for energetic leptons. With $\gamma_{\max }$ determined as the smaller of the limiting values from the two constraints above, the high-energy electron distribution injected at the acceleration site is written as $n_{\text {acc }}(\gamma) \propto \gamma^{-\sigma(p)} \exp \left(-\gamma / \gamma_{\max }\right)$ for $\gamma \gg 1$, where $\sigma$ is the high-energy index of the simulated Monte Carlo distribution $d n / d p \propto p^{-\sigma}$ of accelerated particles.

The radiation module then assumes an equilibrium between the acceleration of particles out to $\gamma_{\max }$ and particle escape out of the radiation zone on a time scale $t_{\mathrm{esc}}=\eta_{\mathrm{esc}} R / c$. If $t_{\mathrm{rad}}<t_{\mathrm{esc}}$, a break in the electron spectra by $\Delta \sigma=$ 1 is expected at a Lorentz factor $\gamma_{b}<\gamma_{\max }$, where the radiative cooling time scale equals the escape time scale. This radiative cooling break is in addition to acceleration flattening breaks illustrated in Fig. 1. The resulting electron spectra are then re-normalized to a kinetic luminosity of electrons in the jet,

$$
L_{\mathrm{kin}}=\pi R^{2} c \Gamma_{\mathrm{jet}}^{2} m_{e} c^{2} \int_{1}^{\infty} n_{e}(\gamma) \gamma d \gamma
$$

which is a free input parameter for fitting purposes. Here $\Gamma_{\text {jet }}$ is the bulk Lorentz factor of the jet. As a change in the electron spectrum (and density) will lead to changes in the co-moving synchrotron photon field, our code determines the equilibrium electron distribution through an iterative process. It starts by considering only the magnetic $\mathcal{U}_{B}$ and external radiation $\mathcal{U}_{\text {rad }}$ energy densities to determine a first-order equilibrium $e^{-}$distribution. Then $\mathcal{U}_{B}$ is used to evaluate the co-moving synchrotron photon field, which is added to the energy densities to re-determine the electron cooling rates and to re-evaluate the break and maximum electron energies. The process is repeated until convergence is achieved, in just a few iterations.

The magnetic field is specified by means of a magnetic partition fraction $\epsilon_{B} \equiv$ $L_{B} / L_{\text {kin }}$, where $L_{B}=\pi R^{2} c \Gamma_{\text {jet }}^{2} \mathcal{U}_{B}$ is the power carried by the magnetic field along the jet, partly in the form of Poynting Flux. The field is assumed to be tangled in the co-moving jet frame, and the radiative output from the equilibrium electron distribution is evaluated using the radiation transfer modules of Boettcher et al. ${ }^{20}$.

\subsection{Multiwavelength Spectral Model for AO $0235+164$}

To illustrate the advances in understanding offered by combining shock acceleration simulation results with radiation emission codes, we study here the low synchrotron peak frequency BL Lac object $\mathrm{AO} 0235+164$ at $z=0.94$. It belongs to a group of extragalactic jet sources with the $\nu F_{\nu}$ spectrum peaking in the optical. However, interestingly, in flaring, it is observed to possess a significant X-ray excess, consistent with the so-called Big Blue Bump (BBB). Such enhancements have been suggested 4 to be a signature of a bulk Comptonization effect, where hot thermal electrons upscatter an ambient quasi-thermal radiation field, perhaps in the infra-red or optical bands. This seed field could be disk-related, or perhaps dust emission possibly from 
the line regions, but is distinct from the non-thermal synchrotron emission. Here, one of the objectives is to explore whether the BBB can be attributed to the substantial thermal electron pool evinced in shock acceleration predictions such as in Fig. 1. In the fast-moving jet, this population may produce significant radiative signatures due to Comptonization of an external radiation field.

The BL Lac object AO $0235+164$ was observed to be flaring in the period during September - November 2008. We focus here on modeling data collected during the multi wavelength campaign discussed in Ackermann et al ${ }^{41}$; see also Agudo et al ${ }^{42}$ for additional flare light curves and polarization data. The list of observatories and missions participating in this campaign is extensive 4 - it spans radio and optical, with Swift XRT and RXTE providing X-ray monitoring, and Fermi-LAT providing the principal gamma-ray observations. The LAT gamma-ray spectrum displays a break or possible turnover at energies around $3-4 \mathrm{GeV}$, with a power-law index of about 2.1 below this feature; the source is not detected in the $\mathrm{TeV}$ band $\frac{43}{4}$. The broadband spectrum for the high state portion of the outburst, during the interval MJD 54761-54763 (October 22-24, 2008) is depicted in Fig. 2 This appears in Fig. 7 of Ackermann et al. 1 , , wherein it can be compared with a low state spectrum obtained about six weeks later that does not contain a Swift XRT detection.

The jet parameters used to derive the model fit include the bulk Lorentz factor of $\Gamma_{\text {jet }}=15$, and the electron kinetic luminosity of $L_{\text {kin }}=1.55 \times 10^{47} \mathrm{erg} / \mathrm{sec}$. The magnetic luminosity $L_{B}$ was a tenth of this so that the field was marginally sub-equipartition: for this one-zone model, $B \approx 0.45$ Gauss was obtained. To place the synchrotron turnover frequency in the optical band $\left(\sim 10^{14} \mathrm{~Hz}\right)$, the values of $\eta\left(p_{\max }\right)$ and $B$ have been chosen so that the maximum electron Lorentz factor in the comoving frame of the jet is $\gamma_{\max } \approx 5.53 \times 10^{3}$, a parameter connecting to the shock acceleration zone. This then placed the peak SSC frequency at around $\gamma_{\max }^{2} 10^{14} \mathrm{~Hz} \sim 10^{21} \mathrm{~Hz}$, i.e. around $10 \mathrm{MeV}$, as is evident in Fig. 2, A cooling break is apparent in the SSC spectrum at around a few keV. Observe that the SSC component is of insufficient luminosity to model the Swift XRT and Fermi-LAT signals - another component is needed to explain them.

Here we model these detections using bulk-Comptonization/inverse Compton scattering of an external radiation field. This field is presumed to be quasi-isotropic in the observer's frame, so that the jet material moves at $\Gamma_{\text {jet }}=15$ relative to these seed photons, and so can inverse Compton scatter them, i.e. increase their frequency by a factor of $\sim 4 \Gamma_{\text {jet }}^{2}$. The minimum Lorentz factor of an electron accelerated in the distributions of Fig. 1 is less than 2, so this adds at most a factor of two to this IC energy enhancement. Hence, to explain the Swift XRT flaring flux, the seed background radiation needed a temperature of $T \approx 6 \times 10^{3} \mathrm{~K}$, with a Planck energy density of $\mathcal{U}_{\mathrm{rad}}=1.1 \times 10^{-5} \mathrm{erg} \mathrm{cm}^{-3}$. This corresponds to optical radiation, perhaps from the accretion disk. The thermal portion of the electron population boosts the ambient photons up to the X-ray range, and roughly produces the steep Swift XRT spectrum if it corresponds to the upper side of the Maxwell- 


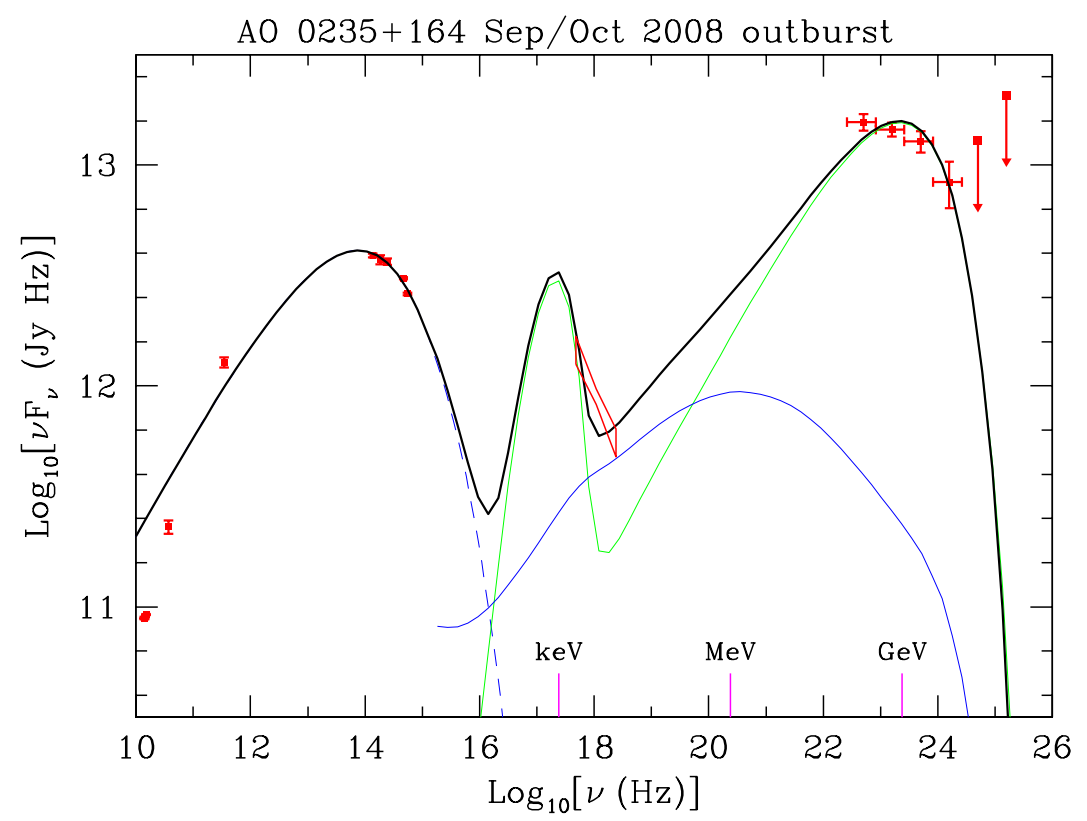

Fig. 2. Multiwavelength $\nu F_{\nu}$ spectra (points) spanning the radio, optical, X-ray and gammaray bands, together with model fits as described in the text, for the September/October 2008 flaring event of the blazar AO 0235+164. The campaign data are taken from Ackermann et al. ${ }^{41}$, displaying a high state epoch for October 22-24. The gamma-ray detections and upper limits are from Fermi-LAT, while the X-ray "butterfly" block represents Swift XRT data. The broadband models consist of a synchrotron component (dashed blue curve) up to the optical band, an SSC component in the X-rays and gamma-rays (solid blue curve), bulk Comptonization emission (green solid curve), totaling the black spectrum. The data and spectral model constrained the diffusion parameters to $\eta_{1}=15$ and $\alpha=3$.

Boltzmann distribution. This is clearly evident in Fig. 2. This then connects to a very flat external Compton tail, generated by the broken power-law tail portions of the distributions depicted in Fig. 1. modulo the cooling breaks discussed above. This IC tail possesses a spectral index of around $3 / 2$, rising in the $\nu F_{\nu}$ representation to meet the Fermi-LAT flux above $100 \mathrm{MeV}$. The details of the various spectral components are outlined at greater length in Böttcher et al $\stackrel{44}{4}$. The key acceleration/diffusion parameters for the fit will be discussed below. To leading order, this one-zone hybrid acceleration/radiation model describes the multi wavelength spectrum of $\mathrm{AO} 0235+164$ in its high outburst state, but only using a contribution of external-radiation Comptonization from the jet material, a conclusion similar to that in Ackermann et al 41 . 


\section{Discussion}

The multiwavelength spectral fits obtained here using full electron distributions derived from diffusive shock acceleration simulations enable useful diagnostics on the plasma environment in blazar jets. The key constraints derived are representative values of $\eta(p)=\lambda_{\|} / r_{g}$ at the low injection momenta, $p \sim p_{1}$, and at the maximum momentum $p_{\max }$ in the shock acceleration zone. These two mean free path parameters couple via the diffusion index $\alpha$. Here we focus first on $\eta\left(p_{\max }\right)$, which for our blazar case study far exceeds unity. The reason for this is that it is required to fit the frequency of the synchrotron $\nu F_{\nu}$ peak just below the spectral turnover of this emission component. For strong cooling in the acceleration zone, the turnover is created when the cooling rate $-d \gamma / d t=4 \sigma_{\mathrm{T}} \mathcal{U} \gamma^{2} /\left(3 m_{e} c\right)$ is approximately equal to the particle acceleration rate $d \gamma /\left.d t\right|_{\text {acc }} \sim\left(u_{1 x} / c\right)^{2} e B /(\eta m c)$ in the Fermi process. Now introduce a cooling parameter $\epsilon_{\text {syn }}=\mathcal{U}_{B} / \mathcal{U}$ that represents the fractional contribution of the synchrotron process to the electron cooling. This is approximately $\epsilon_{\mathrm{syn}} \approx L_{O} / L_{\gamma}$, which has a value of about $0.12-0.15$ according to the broadband spectrum in Fig. 2. If $\epsilon_{\mathrm{syn}} \gtrsim 1$, then this acceleration/cooling equilibrium establishes the well-known result that $\gamma_{\max } \propto B^{-1 / 2}$ and the synchrotron cutoff frequency is independent of the magnetic field strength:

$$
E_{\mathrm{syn}} \sim \frac{\Gamma}{\eta}\left(\frac{u_{1 x}}{c}\right)^{2} \frac{m_{e} c^{2}}{\alpha_{\mathrm{f}}}
$$

Here $\alpha_{\mathrm{f}}=e^{2} /(\hbar c)$, and the blueshift factor $\Gamma \equiv \Gamma_{\text {jet }}$ due to Doppler beaming has been included. This result holds provided that the acceleration process is gyroresonant, which is the prevailing paradigm for both non-relativistic and relativistic shocks. For $\Gamma=1$ and $u_{1 x}=c$, the $\eta\left(p_{\max }\right)=1$ Bohm limit of this is around $100 \mathrm{MeV}$, as was highlighted in De Jager et al $\stackrel{45}{5}$ for considerations of $\gamma$-ray emission at relativistic pulsar wind nebular shocks. For $\epsilon_{\text {syn }}<1$, as is the case for AO $0235+164, p_{\max }$ becomes weakly dependent on $B$, and the turnover energy $E_{\text {syn }}$ drops somewhat below the estimate in Eq. (3), and becomes proportional to $B$.

For our application to $\mathrm{AO} 0235+164$, we set $\Gamma \equiv \Gamma_{\text {jet }}=15$ and $u_{1 x} / c=0.71$. To move $E_{\text {syn }}$ into the X-ray or optical bands, one requires very large values for $\eta\left(p_{\max }\right)$, thereby dramatically reducing the rapidity of the acceleration process. This was the approach of Inoue \& Takahara ${ }^{\sqrt{19}}$, who chose $\eta \sim 10^{5}$ when exploring multiwavelength modeling of Mrk 421 spectra. For the AO 0235+164 fitting protocol here, one needs much larger values $\left(\eta \sim 10^{8}\right)$ to place $E_{\text {syn }}$ in the optical band. Values this large suppress injection of thermal charges into the acceleration process almost completely $y$. Therefore the path to maintaining efficient injection but slowing acceleration out to the maximum momenta is to demand that $\eta$ not be constant, but rather a strongly increasing function of $\gamma$. This was adopted in the fits of Fig. 2, where $\eta_{1}=15$ and $\alpha=3$ (i.e. $\eta(p) \propto p^{2}$ ) yields a synchrotron turnover in the optical. Such constraints on the shock turbulence parameters provide a strong Maxwell Boltzmann component to the electron distribution, as in Fig. 1 and this generates a bulk Comptonization peak in the X-ray band that accommodates the 
Swift XRT spectrum and flux. At the same time, since $\eta(p) \gg 10$ for most electron momenta, the distribution tail quickly asymptotes to a power law with an index $\sigma(p) \sim 1$, corresponding to very active shock drift acceleration in weakly turbulent fields, as discussed above. This then generates the $3 / 2$ photon index evident in the non-thermal external Compton component, when the population is subjected to strong Compton and synchrotron cooling outside the acceleration zone. Since AO $0235+164$ does not have a $\mathrm{TeV}$ detection, its inverse Compton peak energy is low enough to preclude a determination of $\sigma\left(p_{\max }\right)$ using the Fermi-LAT instrument.

The multi-wavelength fits indicate that the physical environment of the shock acceleration region should possess weakening turbulence at larger distances from the discontinuity, driving longer diffusive mean free paths for electrons of larger momenta. This is not entirely without precedent. In the heliosphere, the solar wind contains traveling interplanetary shocks, at which accelerated ions are clearly detected. The turbulence near the shocks is observed to satisfy $\langle\delta B / B\rangle \sim 0.1$, and the diffusive transport of $\mathrm{H}$ and $\mathrm{He}$ ions is inferred to be not very different from the Bohm limit 46 . In contrast, away from these shocks, the turbulence is much more muted, and the diffusive mean free paths are much large ${ }^{31 / 47}$. From this, $\alpha>1$ circumstances must clearly exist. Actual magnetometer measurements ${ }^{48}$ by the Wind spacecraft of the quiet solar wind over a period of several years establishes an inertial range for turbulence $\left\langle(\delta B)^{2} / 8 \pi\right\rangle$ spanning about 3-4 decades in frequency above $\sim 3 \times 10^{-5} \mathrm{~Hz}$. The spectrum for this range is fairly close to the standard Kolmogorov $\nu^{-5 / 3}$ form. Below this frequency, the turbulence spectrum flattens, and one anticipates that diffusion in solar wind plasma turbulence must decline.

From the theoretical standpoint, given a finite inertial (i.e. active) range of turbulence, diffusive mean free paths $\lambda_{\|}$can be estimated ${ }^{\sqrt[31]{1}}$ using quasi-linear theory (QLT). For Kolmogorov-like turbulence spectra, one can quickly arrive at $\lambda_{\|} \propto p^{2 / 3}$, approximately. However, for charges with high momenta and therefore large gyroradii, the Doppler resonance condition $\omega \equiv k c=e B /(\gamma m c)$ is not satisfied by turbulence in the inertial range, only by low frequency waves. Therefore, it can be simply demonstrated that the mean free paths become more strongly dependent on $p$ using the formalism o ${ }^{31}$, namely that approximately $\lambda_{\|} \propto p^{2}$, i.e. $\alpha \sim 2$ and $\eta \propto p$. The diffusion of charges then receives a substantial contribution from non-gyroresonant collisions with inertial turbulence. Such is expected to be the situation for electrons with large gyroradii being accelerated in blazar shocks. Moreover, if the shock-generated inertial range turbulence does not persist at the same levels out to very large distances from blazar shocks, but instead declines in intensity, one can anticipate that $\alpha$ can effectively rise above two. In other words, $\alpha>1$ scenarios are entirely realistic for blazar multi wavelength models. Accordingly, the broadband spectral modeling of AO $0235+164$ presented here provides interesting diagnostics on the nature of plasma turbulence in its shocked jet. 


\section{Conclusion}

This paper has offered a detailed exploration of multi-wavelength spectral fits to flaring emission from the BL Lac object AO 0235+164 using electron distribution functions obtained directly from Monte Carlo simulations of diffusive acceleration at relativistic shocks. This cohesive application of simulated acceleration distributions to blazar spectral modeling provides essentially unprecedented insights into the plasma environment of extragalactic jets. Since shock acceleration feeds off a prominent thermal population of charges, low synchrotron "peak" frequency sources like AO $0235+164$ should possess strong bulk Comptonization signatures from this thermal component. Such is the case here in Fig. 2, accounting for the high-state Swift XRT signal. At the same time, in order to model both the low synchrotron turnover energy in the optical, and the prominent hard gamma-ray flux seen by Fermi, models need the diffusive mean free path to increase rapidly with electron momentum, $\lambda_{\|} \propto p^{\alpha}$ with $\alpha \gtrsim 2$, starting with fairly modest values $\lambda_{\|} / r_{g} \sim 10-30$ at low thermal momenta. We argue here that this strong momentum dependence of $\lambda_{\|} / r_{g}$ is not unexpected, due to somewhat constrained inertial ranges of plasma turbulence, associated non-gyroresonant diffusion, and perhaps also a decline in the level of such turbulence away from shocks that inject energetic particles into the much larger blazar jet emission zones.

\section{Acknowledgments}

MGB and MB are grateful to NASA for partial support for this research through the Astrophysics Theory Program, grant NNX10AC79G. MGB also acknowledges support from the Department of Energy under grant DE-SC0001481. This work is based on research supported by the South African Research Chairs Initiative (grant

no. 64789) of the Department of Science and Technology and the National Research Foundation of South Africa.

\section{References}

1. Hartman, R. C., Bertsch, D. L., Fichtel, C. E., et al. ApJ Lett. 385, L1 (1992).

2. Lin, Y. C., Bertsch, D. L., Chiang, J., et al. ApJ Lett. 401, L61 (1992).

3. Punch, M., Akerlof, C. W., Cawley, M. F., et al. Nature 358, 477 (1992).

4. Dermer, C. D., Schlickeiser, R. \& Mastichiadis, A., Astr. Astrophys. 256, L27 (1992).

5. Sikora, M., Begelman, M. C. \& Rees, M. J., ApJ 421, 153 (1994).

6. Maraschi, L., Celotti, A. \& Ghisellini, G., ApJ Lett. 397, L5 (1992).

7. Mastichiadis, A. \& Kirk, J. G., Astron. Astrophys. 320, 19 (1997).

8. Chiang, J. \& Böttcher, M., ApJ 564, 92 (2002).

9. Abdo, A. A., Ackerman, M., Ajello, M., et al., ApJ 707, 1310 (2009).

10. Abdo, A. A., Ackerman, M., Ajello, M., et al., ApJ 715, 429 (2010).

11. Blandford, R. D. \& Eichler, D. Phys. Rep. 154, 1 (1987).

12. Drury, L. O'C., Rep. Prog. Phys. 46, 973 (1983).

13. Jones, F. C. \& Ellison, D. C. Space Sci. Rev. 58, 259 (1991). 
14. Böttcher, M., Dermer, C. D. \& Finke, J. D. ApJ Lett. 679, L9 (2008).

15. Ellison, D. C., Jones, F. C. \& Reynolds, S. P., ApJ 360, 702 (1990).

16. Kirk, J. G. \& Heavens, A. F., MNRAS 239, 995 (1989).

17. Ellison, D. C. \& Double, G. P., Astropart. Phys. 22, 323 (2004).

18. Summerlin, E. J. \& Baring, M. G., ApJ 745, 63 (2012).

19. Inoue, S. \& Takahara, F., ApJ 463, 555 (1996).

20. Böttcher, M., Reimer, A., Sweeney, K. \& Prakash, A., ApJ 768, 54 (2013).

21. Bednarz, J. \& Ostrowski, M., Phys. Rev. Lett. 80, 3911 (1998).

22. Baring, M. G., in Proc. of the 26th ICRC, Vol. IV, p. 5, Salt Lake City, Utah, (astro-ph/9910128) (1999).

23. Kirk, J. G., Guthmann, A. W., Gallant, Y. A. \& Achterberg, A., ApJ 542, 235 (2000).

24. Peacock, J. A., MNRAS 196, 135 (1981).

25. Kirk, J. G. \& Schneider, P., ApJ 315, 425 (1987).

26. Niemiec, J. \& Ostrowski, M., ApJ 610, 851 (2004).

27. Gallant, Y. A., Hoshino, M., Langdon, A. B., et al., ApJ 391, 73 (1992).

28. Nishikawa, K.-I., Hardee, P., Richardson, G., et al., ApJ 595, 555 (2003).

29. Sironi, L. \& Spitkovsky, A., ApJ 698, 1292 (2009).

30. Stecker, F. W., Baring, M. G. \& Summerlin, E. J., ApJ Lett. 667, L29 (2007).

31. Forman, M. A., Jokipii, J. R. \& Owens, A. J., ApJ 192, 535 (1974).

32. Ellison, D. C., Möbius, E. \& Paschmann, G., ApJ 352, 376 (1990).

33. Moussas, X., Quenby, J. J., et al. Solar Phys. 140, 161 (1992).

34. Giacalone, J., Burgess, D. \& Schwartz, S. J., in Study of the Solar-Terrestrial System, (Noordwijk: ESA Special Publication), p. 65 (1992).

35. de Hoffmann, F., \& Teller, E., Phys. Rev. 80, 692 (1950).

36. Jokipii, J. R., ApJ 255, 716 (1982).

37. Decker, R. B. \& Vlahos, L., ApJ 306, 710 (1986).

38. Begelman, M. C. \& Kirk, J. G., ApJ 353, 66 (1990).

39. Böttcher, M. \& Chiang, J. ApJ 581, 127 (2002).

40. Sikora, M., Madejski, G. Moderski, R. \& Poutanen, J., ApJ 484, 108 (1997).

41. Ackermann, M., Ajello, M., Ballet, J., et al. ApJ 751, 159 (2012).

42. Agudo, I., Marscher, A. P., Jorstad, S. G., et al., ApJ Lett. 735, L10 (2011).

43. Errando, M., et al. Proc. 32nd ICRC (Beijing, 2011). [arXiv:1110.1028]

44. Böttcher, M., Baring, M. G. \& Summerlin, E. J., in Proc. "Fifth International Meeting on High Energy Gamma-Ray Astronomy," eds. F. A. Aharonian, W. Hofmann \& F. M. Rieger (AIP Conf. Proc. 1505) p. 622 (2012).

45. De Jager, O. C., Harding, A. K., Michelson, P. F., et al., ApJ 457, 253 (1996).

46. Baring, M. G., Ogilvie, K. W., Ellison, D. C. \& Forsyth, R. J., ApJ 476, 889 (1997).

47. Palmer, I. D., Rev. Geophys. Space Phys. 20, 335 (1982).

48. Podesta, J. J., Roberts, D. A. \& Goldstein, M. L., ApJ 664, 543 (2007). 